\title{
Notes on the Freedom Tower. Current issues in Networking (mesh).
}

\author{
Camille Akmut
}

\begin{abstract}
Original research notes on the Freedom Tower by Free Network Foundation. Technical characteristics, as well as social, political and philosophical aspects of a real incarnation of a mesh network. Highlights of shortcomings of the academic literature, textbooks in particular, on mesh networks, peer-to-peer, and related subjects.
\end{abstract}

Keywords: mesh; peer-to-peer; internet; networking; networks 


\section{Introduction : mesh networks, social context}

A surge of interest in mesh networks seemed to have happened in 2012, with multiple publications or projects happening in close succession to one another, building on previous efforts ${ }^{12}$.

This coincided with, and could be explained by the publishing, the previous year, of an official standard for Mesh networking by the IEEE in $2011^{3}$. And, the inclusion of the B.A.T.M.A.N. routing protocol inside the Linux Kernel ${ }^{4}$.

This however is not enough explanation - though it may be to some technologists or computer scientists.

In that year, and the previous, multiple socio-political events had made the use of mesh networks evident, vital, or otherwise necessary. The events of the Arab Spring of 2011 and 2012, during which the fragility of the Internet to censorship was again manifested, with access to social media and SMS communications blocked, and the Occupy Wall Street movement, where real uses of mesh networks were experimented, could be cited as examples.

The years before those had known a trend of either increased concentration of Internet infrastructure or/and increased perception of it. ${ }^{5}$

Failings of the traditional Internet, that can be directly attributed to its consolidated nature, and the centralized architectures it relies on, in particular spying by governments on their citizens, and misuse of user data on a massive scale by big corporations, have come to broad light since.

They were the subjects of vast media coverage from 2013 onward, bringing these modern computer technological subjects - perhaps for the first time and on that scale almost certainly - to very broad audiences. These subjects becoming simultaneously, and inseparably, political and social topics of first importance.

All of these factors and events combined give some explanation as to the surge of interests in technologies such as mesh networks, and as to why people may be looking for and already experimenting with alternatives for the Internet, or other forms of networks altogether.

But, little seemed to have hit back home in the academic literature, textbooks in particular where business as usual seemed to be the standard.

We aim to correct this with this publication, and to give a basis for further explorations, improvements and debate.

And maybe, by the year 2020, their inclusion in the seemingly atemporal

\footnotetext{
${ }^{1}$ Motherboard 2012. Berkman Klein Center 2012. Cook 2012. airberry 2012.

${ }^{2} \mathrm{~A}$ notable such project, which runs in several major German cities, is Freifunk, also responsible for the development of the B.A.T.M.A.N. (routing) protocol among others.

${ }^{3}$ IEEE 802.11s.

${ }^{4}$ Implemented in or as B.A.T.M.A.N. advanced (batman-adv). Kernel 2.6.38.

${ }^{5}$ On Internet and Web consolidation, and their effects, among others, see our previous research. "A User-Defined Web. And, on Systems modification in general."
} 
books of knowledge of these gentlemen - how else do they expected research to move forward on these topics if students do not know about them.

Structured notes on a real incarnation of a mesh network, and its technological characteristics as well philosophical-political aspects follow.

Technical innovations bring or require a transformation of our language - unsure and uncertain yet, but here, and growing. "A "user owned" Internet", "User Owned Communications Infrastructure", "a People's "Internet"”, "Internet-like networks". Powerful ideas cannot be removed so easily.

These efforts have had and continue to have incarnations, in metal, software and paper, and the people that create them, one we discuss here.

In presenting the topic, the Freedom Tower, we have taken the approach to give as much space as possible to 1 . the descriptions of the makers or creators 2. secundary sources connected to the creators (one documentary, one report) 3. the wider available literature of computer science and networks/networking. This includes forgoing a longer introduction (which we do not include in the numbering, neither do we the conclusion), this includes minimizing commentary to the only minimum necessary, and certain, and marking those that lead to digressions accordingly.

The aim of this report can be understood further to be a compact (portable), structured source of information. 


\section{The Freedom Tower}

The "Freedom Tower" by the Free Network Foundation ${ }^{6}$ is an example of such a network, or of how such networks can be created.

One of its directors, Isaac Wilder ${ }^{78}$, described their work as

We build decentralized and distributed communication systems. ${ }^{9}$

They are "independent wifi sources (...) beaming out free, secure Internet" 10 - the narrator comments while the following (animated) schema is shown.

\footnotetext{
${ }^{6}$ Found abbreviated as FNF.

${ }^{7}$ Listed as "executive director" in the documentary Free the Network by Motherboard (Motherboard 2012). The graphics in the figures are extracted from this work.

${ }^{8}$ Another important figure of this project is or was Tyrone Greenfield (son of Jerry Greenfield (Ben \& Jerry's)).

${ }^{9}$ Ibid.

${ }^{10}$ Ibid.
} 


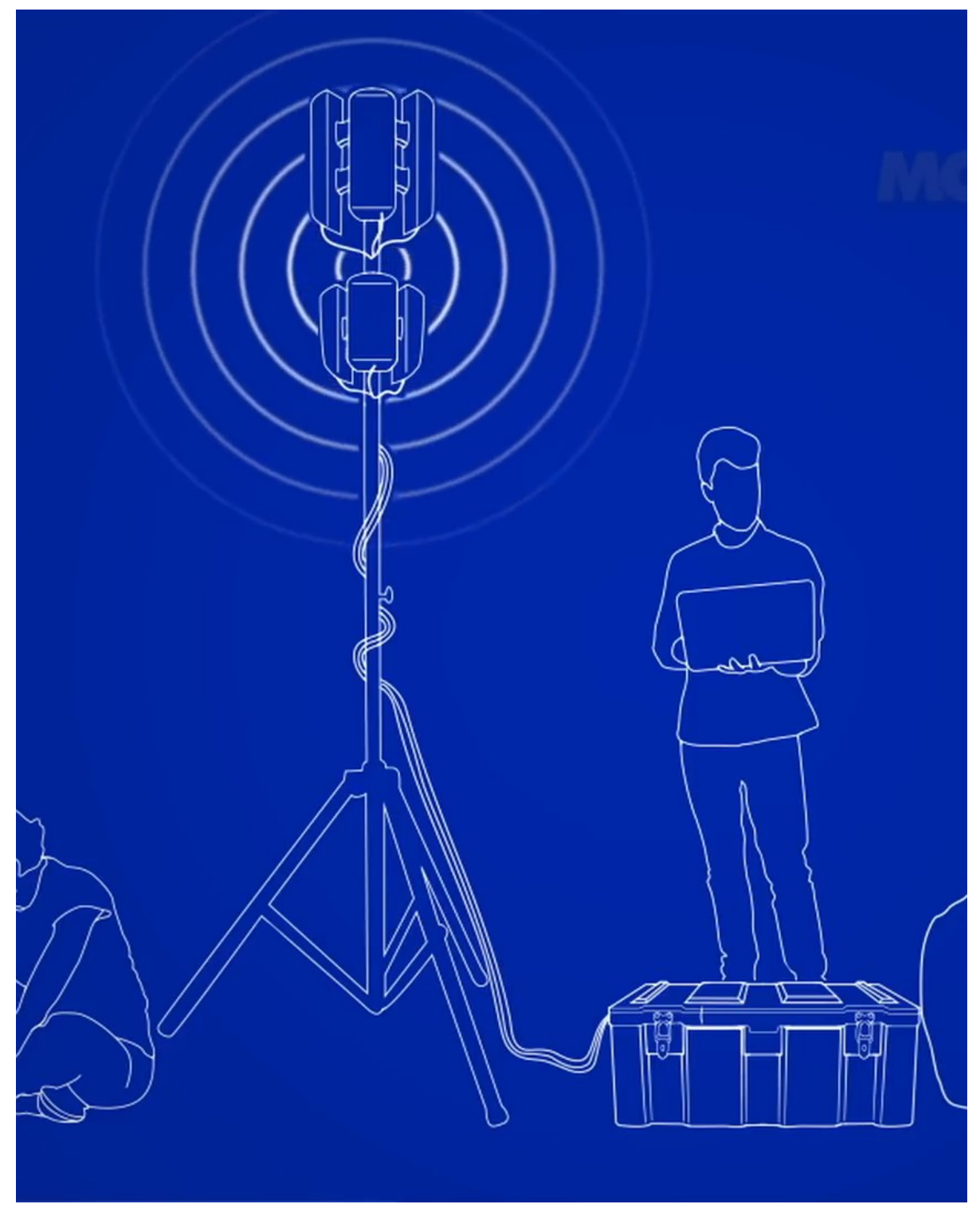

Figure 1: Freedom Tower (Motherboard 2012).

At a high level, of abstraction, it can be said that the Freedom Tower consists of two main components : the enclosure (shown at the bottom right in the graphic of figure 1), and the pole (extending from the bottom to the top, towards the left of the same graphic).

The equipment necessary for these Freedom Towers, hardware and software, is given by Wilder as follows :

There's the modems, the router, and the radios. The modems that we're using connect to Clear [a wireless Internet service provider 
(ISP)]. In the future we'll be able to replace those with modems that connect to our radios, or to anybody's radios. The key I guess, the secret sauce right, is the software that's running on the router, that lets you anonymize, or tunnel, or do these kinds of fancy tricks with network traffic. ${ }^{11}$

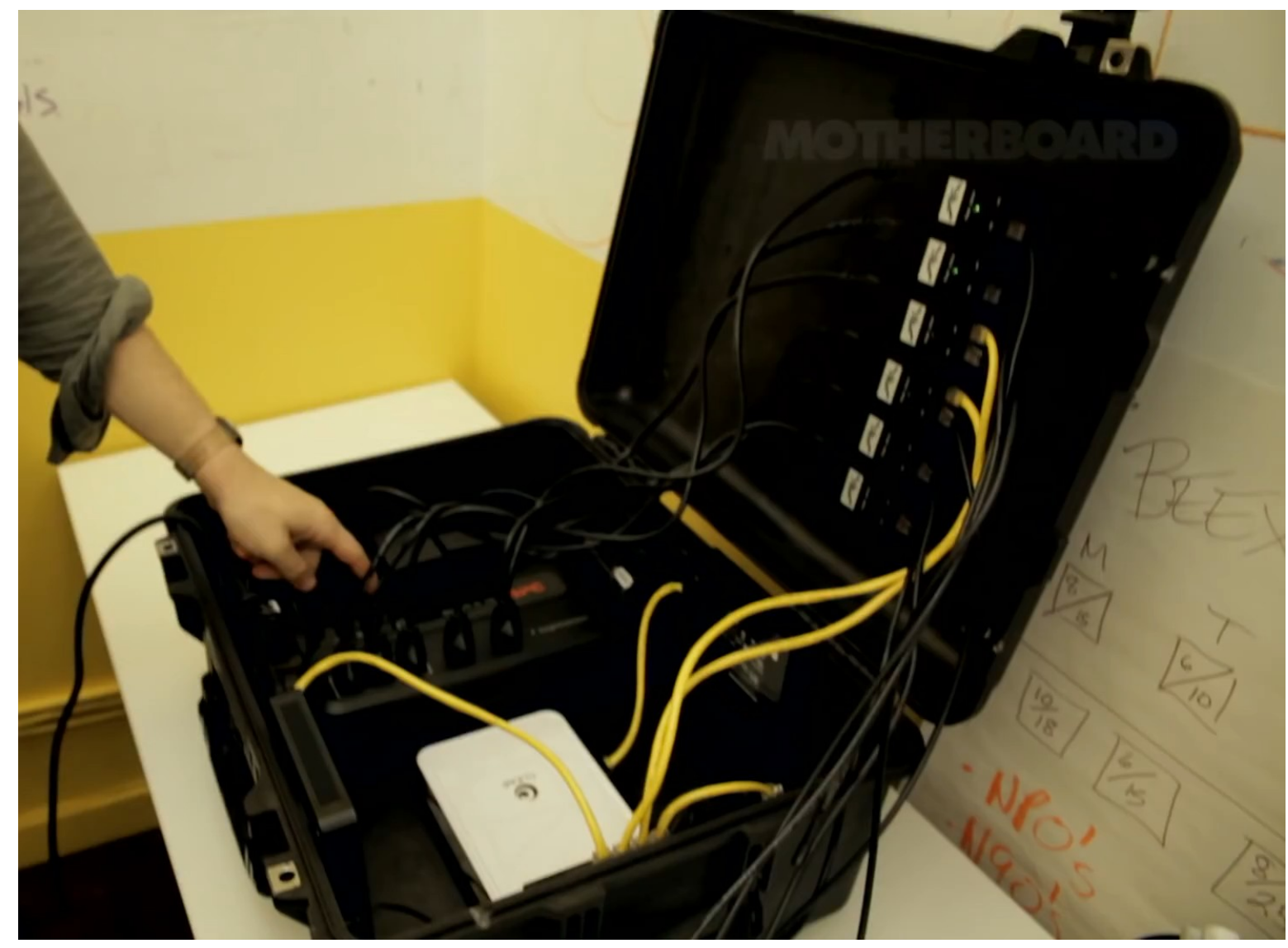

Figure 2: Freedom Tower component.

The "enclosure", or trunk ${ }^{12}$, shown in figure 2 , he describes as :

The router is connected on one side to the wide area network, so that is like the backhaul ${ }^{13}$, or wherever we're getting our upstream

\footnotetext{
${ }^{11}$ Ibid.

${ }^{12}$ For lack of better or official terminology.

13 "Backhaul" is Networking terminology (not to be confused with "back hall"). In Comer, backhaul appears in relationship to WiMAX : "WiMAX offers broadband communication that can be used in a variety of ways. Some service providers plan to use WiMAX as an Internet access technology that spans the last mile. Others see the potential of WiMAX to provide a general-purpose inter-connection among physical sites, especially in a city. Another type of interconnection is known as backhaul - the connection between a service provider's central network facility and remote locations, such as cell towers." (Comer 2015 : 308).
} 
bandwidth from.

You have this separation of components, so that this is modular, and you can replace a WiMAX ${ }^{14}$ [high-speed wi-fi for large geographical areas] modem, with whatever kind of modem, or input that you want.

The wide area connection comes into the router. The router is connected to the switch, which then connects it to the server.

These two connections go to power injectors for the two gateway radios.

One on each ring is the gateway. And, then the other two, connect to that wirelessly, and redistribute the signal in the other directions. (...) any kind of software that we want to run locally, Web software, whatever kind of software, we can run on this server. It's a Debian server. And, it will be accessible whether the wide area connection goes down, stays up, whatever. This has nothing to do with the global Internet. This is available locally. ${ }^{15}$

\footnotetext{
14 "WiMAX : Wireless access technology up to 155 Mbps using radio frequencies" (Comer $2015: 243)$.

${ }^{15}$ Motherboard 2012.
} 


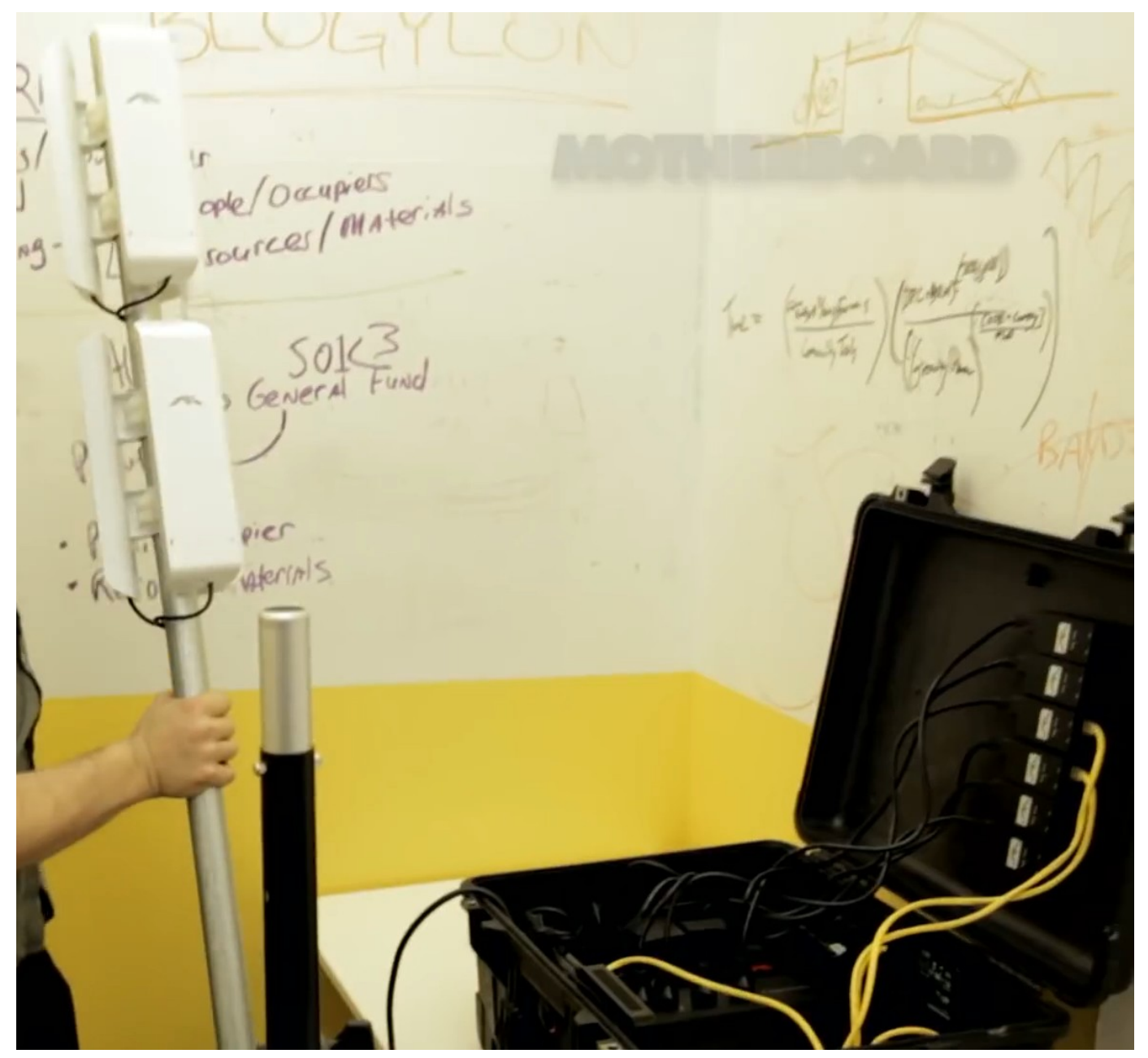

Figure 3: Freedom Tower.

In the report "Peer to Peer User Owned Communications Infrastructure"16, specific details are given ${ }^{171819}$.

It is worth to note, before we move on, that some of the indications in this report - if not all - seem to refer to a (seemingly) more primitive version of

\footnotetext{
${ }^{16}$ Cook 2012
}

${ }^{17}$ In particular, where technical details are concerned, in the section "Freedom Tower Materials and Assembly" (Ibid., p. 29-32).

${ }^{18}$ Of which we cite here only a few, as for the hardware : "- A quiet power generator such as the Honda EU1000i (\$800), - A UPS such as the APC ( ...) (\$100), - A nettop [computer] (...) - An 8-port network switch $(\$ 15)$ - Three USB [to] Ethernet adapters $(\$ 30$ x3) - Two $4 \mathrm{G}$ modems such as the Clear Series M (...) - Three Ubiquiti NSM2 (...) - Three Ubiquiti NSM5 Loco (...)" (Ibid., p. 29).

${ }^{19}$ From our research, we only conclude that the mentioned "radios" are the Ubiquity devices mentioned in their report. The modems being $4 \mathrm{G}$ modems (by Clear), as they appear in that report, also described as WiMAX in the documentary, and the router a computer with router software installed on it, still outgoing from information found in that same report. 
((or) parts of) the Freedom Tower, and as such there may be slight variations in places, which we note whenever we $\mathrm{can}^{20}$. It is not clear if it is a more primitive version, as we assume, and describe here, or an alternative (co-existing) solution, different iteration. ${ }^{21}$.

This seemingly more primitive version of the enclosure, and hence the (corresponding) Freedom Tower (component), than shown here, almost resembles a hard-shell suitcase, with components cluttered inside ${ }^{22} .{ }^{23}$

The contents of the "enclosure" 24 are listed there as follows :

Enclosure for UPS, computer, router, modems, and radio power injectors $^{25}$

We do our best here to identify them in the depiction in figure $2^{26}$ : (directions are given arbitrarily from the point of view of the modem) the modem is the white box, the UPS is above it, the switch (hard to distinguish) is below it, to its right (hard to distinguish) is the router, to its left the server ${ }^{27}$, while the radio power injectors are attached to the lid of the enclosure.

As far as we can tell, in particular based on figure 7, which can be found in the appendix, and offers a better view of the insides, the UPS occupies all of the upper-half of the trunk (i.e. there are no more additional components there).

The outgoing power cable, that can be seen exiting the box, we can only assume goes to a power source : in the office where this was filmed, a regular power outlet we presume, but in the outside, the power generator mentioned in

\footnotetext{
${ }^{20}$ This more "primitive" version is depicted on page 27 of Cook 2012. Because of this, we also include it in our appendix.

${ }^{21}$ The documentary was released on "Mar 29, 2012", while the Cook report features the mention of "March April 2012 Part 2", and its URL contains the date of "2012-04-13", of which a longer part here : “.../2012/04/2012-04-13-Cook-...". For lack of better proposals these two variants may have to be referred to as "Motherboard 2012" and "Cook 2012" (versions), to help facilitate discussion, or narration.

${ }^{22}$ In our opinion, it demonstrates that all such creations have to start somewhere, at a more basic level, from which they can always be improved. Something also found in Huang 2018. Seeing such imperfect states - in actual images, or better photographs - helps us break with fetishizations of technology. (We use this word in more or less the same sense as Marx does, in particular when he talks about the phenomenon of the "fetishization of the object", by which products or goods are disconnected from their origins, the workers. (This is most likely either in Capital, if so most likely in the first book, or Grundrisse.) (Found elsewhere, with some possible inflections, as "commodity fetishism".))

${ }^{23} \mathrm{As}$ for the enclosure, though this is pure speculation on our part, and though various safety considerations would have to be considered, a primitive but widely available alternative could be constituted by a ((slightly) modified) hard-shell suitcase.

${ }^{24}$ Or trunk, we propose for lack of better/official terminology.

${ }^{25}$ Ibid.

${ }^{26} \mathrm{~A}$ much better view is given by the depiction in figure 7 placed in the appendix - minus the lid (where the radio power injectors are).

${ }^{27}$ Which we identify with "computer".
} 
the report, if no other, more practical sources of energy are present (e.g. power outlet).

In further describing the Freedom Tower, we follow the order (given originally by Wilder) : modems, router (software), and radios. And, provide additional information on other components where we think useful.

the modems (we suggest two, but you could use as few as one or as many as are needed) (...) Because all Clear modems are set to the same address by default, it will be necessary to change the DHCP settings so that each modem has a different address. Leave one modem as 192.168.15.1, and number additional modems by iterating the third octet ${ }^{28}: 192.168 .16 .1,192.168 .17 .1$, etc. $^{29}$

As for the router, we deduce from the report that the router is a portable computer on which a Unix-like operating system is installed :

Router The heart of the Freedom Tower is a nettop computer running pfsense, a variant of openbsd, that makes it easy to do network administration. ${ }^{30}$

OpenWrt, a Linux distribution, which supports mesh networking, may represent an alternative ${ }^{3132}$. A laptop may also represent an alternative to the mentioned "nettop" (a small format PC) ${ }^{33}$.

As for the "radios" :

There are 6 radio's overall. Three $2.4 \mathrm{GHz}$ radios and three $5 \mathrm{GHz}$ radios. The $2.4 \mathrm{GHz}$ radios transmit with a strength of $18 \mathrm{~dB}$ a piece and the $5 \mathrm{GHz}$ radios transmitted at the rate of $11 \mathrm{~dB}$ a piece. This gives us a coverage radius of about a half a mile. ${ }^{34}$

If we are correct in their identification with the Ubiquity devices, we can add the following information: The NSM2 model operates on frequency $2.4 \mathrm{GHz}$

\footnotetext{
${ }^{28}$ Octet being the technical term used in Networking to describe the different parts ("numbers") of an IP address : the third octet in the provided example changes from 16 to 17.

${ }^{29}$ Ibid.

${ }^{30}$ Ibid.

31 "OpenWrt can of course run in normal PC or server hardware, and take advantage of the much more powerful hardware the x86 (Intel/AMD) architecture can offer." https:// openwrt.org/docs/guide-user/installation/openwrt_x86.

${ }^{32}$ It has been used, and modified by Freifunk: "The Freifunk Firmware is a modified version of OpenWrt Linux Version that is developed for router devices." https://wiki.freifunk.net/ Kategorie: English

${ }^{33}$ On Linux, in the example we provide specifically Debian though this should be applicable to other distributions as well, the /etc/.../logind.conf file is edited such that HandleLidSwitch=ignore to prevent interruption of a laptop on lid closing (overheating may be a long-term issue of this hence stability). https://wiki.debian.org/Suspend

${ }^{34}$ Cook $2012: 27$.
} 
and the NSM5 on $5 \mathrm{Ghz}$ (as reflected by their nomenclatures) while their range is over 10 kilometers, according to the specifications of the manufacturer ${ }^{35} .{ }^{36}$

These devices are described by the manufacturer as "ideal for Point-toMultiPoint (PtMP) applications" (also found elsewhere as "P2MP" or "PMP").

We provide more information about other components :

Inside the box is an uninterruptible power supply. The UPS power supply will allow the- tower to operate for a couple of hours on battery power alone. ${ }^{37}$

We have followed the nomenclature of "enclosure" (present inside the report) to describe one component of the Freedom Tower. We propose pole ${ }^{38}$ to describe the other (where the radios are) - to make discussion easier.

In the appendix, we include a photo of the Freedom Tower (pole?) in the outside.

To break with the fetishization of electronic or technological equipment, that we may have encouraged so far, that makes it so that they are disconnected from their uses and origins, it is important to note that the Freedom Towers were used at Zuccotti Park, New York, among other places, to support Occupy Wall Street protests by providing protesters "free, secure" wireless Internet access. ${ }^{39}$

Wilder described their goal as follows, under consideration of the system's current - then - limitations :

Some day, our aspiration, is to help humanity build its own actual network. Not a virtual network. But, an actual network. But, in the mean time, a virtual network is the best we can do. And, it's a necessary step in the process of bootstrapping an actual wide area network. ${ }^{40}$

\footnotetext{
${ }^{35}$ https://www.ui.com/airmax/nanostationm/

${ }^{36}$ This is consistent with the following mention in their report : "upstream connectivity is rebroadcast via powerful $2.4 \mathrm{GHz}$ and $5 \mathrm{GHz}$ radios on an open wifi network." (Cook $2012: 5$ ). ${ }^{37}$ Cook 2012 : 27.

${ }^{38}$ A terminology also found in Cook 2012 : "a thin, maybe nine-foot-tall pole, loaded on all sides with nondescript routers that had been beaming out wireless access since early on in the occupation." Either a. this may refer to an alternative or more primitive version of the Tower b. Cook 2012 is wrong when he talks of routers (instead of radios) c. we are wrong (and hence may be wrong in other places, as we identify them as radios) d. some other misconception.

${ }^{39}$ A service that Wilder described we believe as "comfort" to them (in the same way that other people have offered, historically, and presently, food, drinks, water or coffee, or else, and shelters, to protesters, this would be a digital equivalent of that, and one that is beyond that necessary to relay information both locally, to organize, and to the wider world, for information).

${ }^{40}$ Motherboard 2012.
} 
Following this, the narrator of the documentary comments (we assume their work has received some approval from the Free Network Foundation or some of their members at least, which is why these comments are also important or of value to us) :

It's about peer-to-peer networks : people talking to one another, directly, and through no middleman.

The idea is to build up mesh networks, where all points of connection, or nodes, simultaneously receive and release information. But, on top of that, these nodes act as transmitters for other nodes too.

Isaac and the FNF are working to decentralize a global Internet that's become widely consolidated, and to redistribute the avenues we talk to one another.

Mesh networking is not a new idea. It's been spreading around the world in recent years, a sort of pirate radio Internet, that connects underserved communities to the Web $(\ldots)^{41}$

Meanwhile this animated schema is shown :

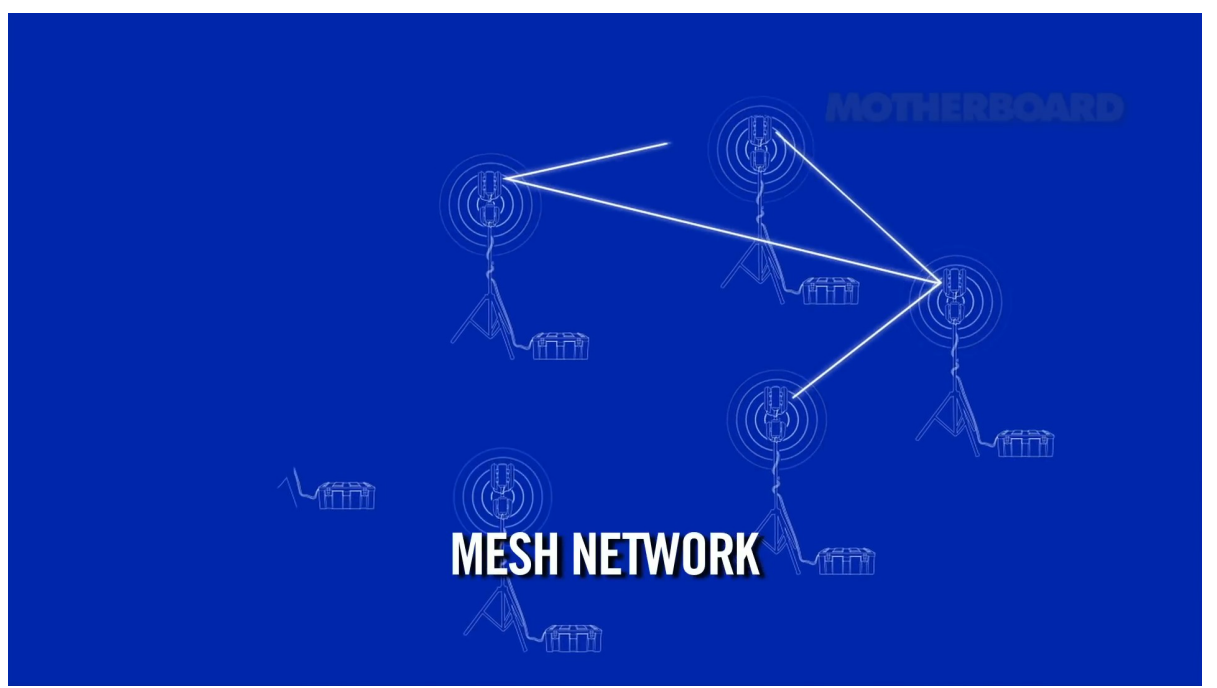

Figure 4: Freedom Towers in a mesh network (Motherboard 2012).

In this animated schema, which we are not able to render fully here, other than in words, links are successively established between the various Freedom Towers ${ }^{42}$, while new Towers emerge, or are created, who are then in turn linked to the (mesh) network.

\footnotetext{
${ }^{41}$ Ibid.

${ }^{42}$ This we assume are the "nodes" mentioned in the narration of the voice-over speaker.
} 
This goes on until the animation stops with the depicted mesh network in the following state : 6 Freedom Towers in total, where each Tower has at least links with two other Towers (their next closest Towers from what we are able to tell). But, some Towers, 4 in total, have links with more than two towers : they are (all) linked to three Towers in total.

This represents a classic graph problem, and fits into the wider topic of graphs (and graph theory) : nodes are connected to each other through socalled edges (or vertices).

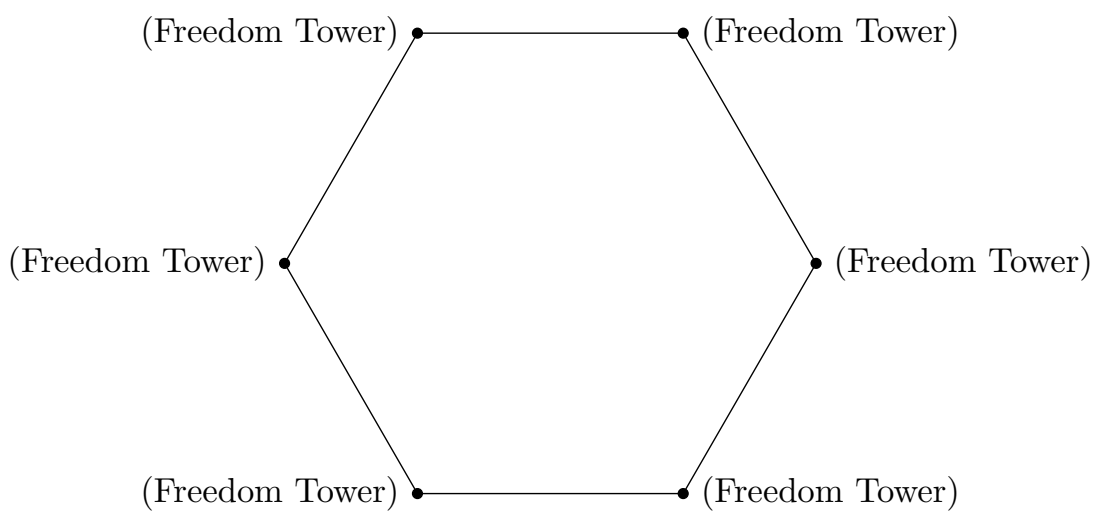

Figure 5: -

Graph representations and problems have (long had) applications in networking, in particular shortest-path algorithms e.g. Dijkstra's algorithm or, maximum flow, Ford-Fulkerson algorithm.

Applied to the final state of the depicted mesh network, this means 6 nodes and 9 edges ( 6 edges on the outside, forming a hexagon of sorts, plus 3 traversal ones on the inside).

The idea of peer to peer (networks or network) is also reflected in the title of the report cited previously, "Peer to Peer User Owned Communications Infrastructure" (containing various materials written in cooperation with, and to 
the best of our knowledge partly, by them).

Specifically, talk is of so-called "Mesh networking" (also found elsewhere as "mesh network", or "meshnet").

The goal is given by the narrator of the documentary as decentralization (of the Internet). ${ }^{43}$

In the academic literature, a definition is given as follows for peer-to-peer architecture :

"In a P2P architecture, there is minimal (or no) reliance on dedicated servers in data centers. Instead the application exploits direct communication between pairs of intermittently connected hosts, called peers. The peers are not owned by the service provider, but are instead desktops and laptops controlled by users (...). Because the peers communicate without passing through a dedicated server, the architecture is called peer-to-peer." ${ }^{44}$

This model is in contrast with so-called "client-server architecture", where "an always-on host, called the server, (...) services requests from many other hosts, called clients." 45

In other places, the terminology "hub and spoke" (architecture) is used (instead).

To understand mesh networks, we must understand how they're different from typical network architectures, used to connect our devices, such as phones, computers and routers. These devices usually connect in what is referred to as a "hub and spoke network architecture". Hub and spoke networks are centralized networks, in which points on the network - the "spokes" - are all connected to a single center - the "hub", which serves as an access point through which all devices on the network connect to all other devices.

In a mesh network, the network architecture is not as defined rather, the devices on the network connect to all other devices on the network that are nearby. The devices, also known as nodes, send data to other nodes that are within range, and the data moves through the network, node to node, until it reaches its final destination.

\footnotetext{
${ }^{43}$ This represents an alternative to the phenomenon of concentration of Internet infrastructure and consolidation of the Internet as a product owned, by few, rather than a "good", by, and for many. Rather than the Internet being owned by a dozen or so of private companies, with for-profit interests, this would constitute a People's "Internet".

${ }^{44}$ (Kurose and Ross 2017 : 114-115).

45 (Ibid.).
} 
But, what are these nodes? They can take many forms as long as they can send and receive data. Typically, they're made of wireless routers. Just like the ones you use at home. They can also be made of cell phones or radios.

For a mesh network to work, however, each node has to behave a bit differently than it would if it were connected to a typical network. It must not only send and receive data, but it must also determine an efficient way to send data across the mesh.

The nodes accomplish this, through what is known as routing protocol.

A routing protocol is a set of rules that are coded into the nodes, which uses either the local, or global make up of the mesh to determine the best path by which to send the data. There are various algorithms that are used for this ${ }^{46}$

To use the terminology of client-server architecture, the hub is the server, and the spokes are the clients.

The term "nodes", used here in the context of mesh networks, most likely coincides with, if it is not replaceable by, the other term, employed by Kurose and Ross in their explanation of peer-to-peer architecture, "peers".

While the documentary does not go into these finer points (of routing protocols), the report does.

"At present, there are two leading algorithms in the arena of mesh routing - Optimized Link State Routing, and the Better Approach to Mobile Ad Hoc Networking. (...) Optimized Link State Routing, or OLSR, is widely utilized. (...) Though recent iterations have decreased CPU usage, and improved throughput, OLSR's primary drawback is heavy CPU usage, especially in discovering and repairing routes. The Better Approach to Mobile Ad Hoc Networking, or BATMAN, emerged from the German FreiFunk community. Its latest iteration, BATMAN Advanced, works at a lower level of the network stack than other mesh implementations, and has now been incorporated into the mailine linux kernel." 47

${ }^{46}$ Berkman Klein Center 2012. This resource goes on to mention "OLSR, Batman, and HWMP."

${ }^{47}$ Cook $2012: 43$. 


\section{Mesh networks (Shortcomings of academic lit- erature)}

In Kurose and Ross, mesh networks appear in two places ${ }^{48}$.

A "mesh networks, wireless" ("wireless mesh networks") entry in the index, itself referring to page 552, which is part of chapter 6, "Wireless and Mobile Networks", in a passage where a "taxonomy" of the "different types of wireless networks" are given

At the highest level we can classify wireless networks according to two criteria: (i) whether a packet in the wireless network crosses exactly one wireless hop or multiple wireless hops, and (ii) whether there is infrastructure such as a base station ${ }^{49}$ in the network:

Single-hop, infrastructure-based. These networks have a base station that is connected to a larger wired network (e.g., the Internet). (...)

Single-hop, infrastructure-less. In these networks, there is no base station that is connected to a wireless network. However, as we will see, one of the nodes in this single-hop network may coordinate the transmissions of the other nodes. (...)

Multi-hop, infrastructure-based. In these networks, a base station is present that is wired to the larger network. However, some wireless nodes may have to relay their communication through other wireless nodes in order to communicate via the base station. Some wireless sensor networks and so-called wireless mesh networks fall in this category.

Multi-hop, infrastructure-less. There is no base station in these networks, and nodes may have to relay messages among several other nodes in order to reach a destination. Nodes may also be mobile,

${ }^{48}$ The other being a passage out of "6.3.6 Personal Area Networks: Bluetooth and Zigbee" on Zigbee, an alternative wireless network protocol/standard to Bluetooth : "Nodes in a Zigbee network come in two flavors. So-called "reduced-function devices" operate as slave devices under the control of a single "full-function device," much as Bluetooth slave devices. A full-function device can operate as a master device as in Bluetooth by controlling multiple slave devices, and multiple full-function devices can additionally be configured into a mesh network in which full-function devices route frames amongst themselves." (Kurose and Ross 2017 : 578).

49 "A base station is responsible for sending and receiving data (e.g., packets) to and from a wireless host that is associated with that base station. A base station will often be responsible for coordinating the transmission of multiple wireless hosts with which it is associated. When we say a wireless host is "associated" with a base station, we mean that (1) the host is within the wireless communication distance of the base station, and (2) the host uses that base station to relay data between it (the host) and the larger network. Cell towers in cellular networks and access points in 802.11 wireless LANs are examples of base stations. (...) the larger network (e.g., the Internet (...) or telephone network)" (Kurose and Ross 2017 : 550). 
with connectivity changing among nodes-a class of networks known as mobile ad hoc networks (MANETs). If the mobile nodes are vehicles, the network is a vehicular ad hoc network (VANET). As you might imagine, the development of protocols for such networks is challenging and is the subject of much ongoing research. ${ }^{50}$

But, no more is said on these topics ${ }^{51}$, and the topic of (wireless) mesh networks specifically, as these authors prefer to "confine [them] selves to singlehop networks, and then mostly to infrastructure-based networks." - leaving the reader to consult some hypothetical research during hours of their own.

In the latest version of Networks, by Tanenbaum and Wetherall, copyrighted 2011, mesh networks only appear once by name, and here also, briefly, only to refer to further research to which the reader may then turn to if their curiosity so desires.

A particularly challenging setting is a wireless mesh network in which multiple, interfering wireless links must be crossed, routes change due to mobility, and there is lots of loss. Research in this area is ongoing. See Li et al. (2009) for an example of wireless transport protocol design. ${ }^{52}$

This is all mesh networks deserve, as envisioned by Tanenbaum and Wetherall (as part of an aptly named "Wireless Issues", that here is conferred the unintended double meaning of Issues in Networks teachings, or indeed, Current Issues in Networking). ${ }^{53}$

This is all the coverage undergraduate and graduate students - the public explicitly targeted - will ever receive on these topics, if their schools had picked them, when reading these books. They are the two most assigned textbooks in their domain ${ }^{54}$.

\footnotetext{
${ }^{50}$ Kurose and Ross 2017 : 552-553.

${ }^{51}$ Except "Hosts associated with a base station are often referred to as operating in infrastructure mode, since all traditional network services (e.g., address assignment and routing) are provided by the network to which a host is connected via the base station. In ad hoc networks, wireless hosts have no such infrastructure with which to connect. In the absence of such infrastructure, the hosts themselves must provide for services such as routing, address assignment, DNS-like name translation, and more." (Kurose and Ross 2017 : 550-551)

${ }^{52}$ Tanenbaum and Wetherhall 2011 : 539. Li et al. 2009 is "Block-Switched Networks: A New Paradigm for Wireless Transport".

${ }^{53}$ Their references stop with the year 2010. For the IEEE 802.11s standard, Mesh Networking, the date 2011 is provided by Beard and Stallings, Wireless Communication Networks and Systems (2016).

${ }^{54}$ By far, Kurose and Ross is the most assigned textbook in "Networking" (with a cumulative count of 197, this is as opposed to 3 for the next most-assigned book (in that category) Tanenbaum is the most assigned book in "Networks" (with a count of 138), followed by Peterson's Computer Networks: A Systems Approach (count 132) and Comer's Computer Networks and Internets (41). This is based on the "Open Syllabus Explorer" database, http://explorer.opensyllabusproject.org/
} 
It is difficult to see how in such conditions they would be moved to move into research on them.

An example of the subtle ways in which, in computer science, and other disciplines, some topics, though presented "in general", are given differentiated treatments.

Computer Networks, 5/e is appropriate for Computer Networking or Introduction to Networking courses at both the undergraduate and graduate level in Computer Science, Electrical Engineering, CIS, MIS, and Business Departments.

Tanenbaum takes a structured approach to explaining how networks work from the inside out. $(\ldots)^{55}$

Though, we are certain, and already assured, that Tanenbaum and Wetherall, and Kurose and Ross, and those who use their books, make up for it in their teachings, the consistently infallible explanation for such shortcomings - "it is handled in class", "it is only the basis for "further explorations"". But, maybe these explorations, and secret closed door teachings, ought to be put in writing, just like the rest of the thousand pages of their books.

In Comer's Computer Networks and Internets, mesh networks are strangely enough relegated to a chapter on "The Internet Of Things", the second last chapter of their book - building up the impression that these are all the uses such networks could have and this is where they belong. Mostly, as part of developments on Bluetook and Zigbee.

Even more confusing, the only general treatment we get in this book is a short explanation of mesh topology as part of "Chapter 13 Local Area Networks: Packets, Frames, And Topologies", "13.8 LAN Topologies" :

A network that uses a mesh topology provides a direct connection between each pair of computers. The chief disadvantage of a mesh arises from the cost: a mesh net- work connecting n computers requires:

$$
\text { connections in a mesh network }=\frac{n !}{(n-2) ! 2 !}=\frac{n^{2}-n}{2}
$$

The important point is that the number of connections needed for a mesh network grows faster than the number of computers. Because physical connections are expensive, few wired LANs employ a mesh topology. ${ }^{56}$

\footnotetext{
${ }^{55}$ Description.

${ }^{56}$ Comer 2015 : 228.
} 
It would take any student, undergraduate or graduate, much capacity for imagination to - out of these two disparate treatments of either IoT or LAN put together a notion of mesh networking as applied to the general, wireless, uses described here.

Of peer to peer networks, we also mostly only learn in Comer that "Instead of fetching a complete file from a central server (...) each client that obtains a piece of the file agrees to act as a server and supply the piece to other clients." 57 As for our concerns, many of the developments in this book are stupefying. The other uses that these topologies have, none of which however any reader would find anywhere here, are either forgone or seemingly unknown to these authors. ${ }^{58}$ an upside down pudding recipe for their subject, ins, and digression with digression from check digression transgression with sole transgression academic norm to have created an upside down pudding, instead of

These "experimental" network architectures will remain "experimental" as long as the authors of these textbooks - "widely used and acclaimed" if textbook publishing marketing is to be trusted - will not treat them; and neither should they be widely acclaimed, and even less so used.

A loss for all as these topics arguably represent some of the most exciting and fascinating parts of their domain. ${ }^{59}$

Not only that, but mesh networks have vital uses : in cases of natural disasters, that may severe important Internet links or phone lines, or mademade disasters (e.g. wars), that may have the same effects. But, furthermore, they have been used to provide access to the Internet to underserved places and communities, either rural and/or of often low socio-economic profiles. In addition, they provide means of communication in censorship contexts ${ }^{60}$.

${ }^{58}$ In Kurose and Ross, peer to peer is also mostly only treated from the point of view of file sharing, or distribution, but a broader context for other uses, such as the ones discussed here, to say nothing of their social or political implications, are completely absent (even more surprising as Ross is presented as someone whose "research interests are in privacy, social networks, peer-to-peer networking", little of which is felt however when reading this mostly generic textbook whose only minor quirk, its only transgression of academic tradition, may have been to have created a recipe for an upside down pudding, instead of a straight pudding, and that is not much).

${ }^{59}$ Due to the nature of the topic, it seemed best - as alternative - to look for specialized books on "wireless networks" or "wireless networking", or "wireless communication", as opposed to textbooks on networks or networking in general, but most of these books (e.g. reference works such as Fundamentals of Wireless Communication by Tse and Viswanath or Wireless Communications by Goldsmith) are 15 years old. (Several books and resources handle these topics in more detail and we list them in the bibliography.

${ }^{60}$ Currently, still, at least 1.4 billion people only know a censored version of the Internet we hold for granted : this is the case for the inhabitants of China (whose access is filtered through the so-called "Great Firewall"). During the Arab Spring, several social websites were blocked in addition to communication by SMS, and censorship of traditional media. Mesh networking chat applications were used during the Hong Kong protests of 2014 (Hu 2014).
} 


\section{Routing protocols : B.A.T.M.A.N., OLSR}

An overview of available algorithms is given by the report we cited before : it mentions OLSR and B.A.T.M.A.N. as the two leading algorithms, highlighting the former's wide use and weaknesses (CPU usage), and the inclusion of the latter in the form of Batman advanced in the Linux kernel ${ }^{61}$. Another resource mentioned both as well, and HWMP, Hybrid Wireless Mesh Protocol, $\operatorname{additionally~}^{62}$.

These algorithms are not mentioned anywhere in the academic literature we have had access to (Kurose and Ross 2017, Comer 2015, Tanenbaum and Wetherhall 2011, Tanenbaum alone, etc.), and there can no hope of finding them there any time soon. ${ }^{63}$

The "main development website" for B.A.T.M.A.N. gives the following description :

B.A.T.M.A.N. (better approach to mobile ad-hoc networking) is a routing protocol for multi-hop ad-hoc mesh networks. ${ }^{64}$

If Kurose and Ross' taxonomy of wireless networks was to be used ${ }^{65}$, this would place B.A.T.M.A.N. applications in the "Multi-hop" category, either Multi-hop infrastructure-less (ad-hoc) or infrastructure-based ((wireless) mesh networks)?

In that same - official or as much as we are aware of so - source, B.A.T.M.A.N. advanced is presented :

B.A.T.M.A.N. advanced (often referenced as batman-adv) is an implementation of the B.A.T.M.A.N. routing protocol in form of a linux kernel module operating on layer $2 .{ }^{66}$

In the documentation for the Linux kernel, appearing as "Batman advanced", in "batman-adv", it is documented like so :

Batman advanced is a new approach to wireless networking which does no longer operate on the IP basis. Unlike the batman daemon, which exchanges information using UDP packets and sets routing tables, batman-advanced operates on ISO/OSI Layer 2 only and uses and routes (or better: bridges) Ethernet Frames. It emulates

\footnotetext{
${ }^{61}$ Cook 2012 : 43.

${ }^{62}$ Berkman Klein Center 2012.

${ }^{63}$ These gentlemen may be waiting for an IEEE certification still, or some equivalent, after which point they will apply a puffer of 5 to 10 years before they can turn to them.

${ }^{64}$ https ://www.open-mesh.org/projects/open-mesh/wiki

${ }^{65}$ Kurose and Ross 2017.

${ }^{66}$ https://www.open-mesh.org/projects/batman-adv/wiki/Wiki
} 
a virtual network switch of all nodes participating. Therefore all nodes appear to be link local, thus all higher operating protocols wont be affected by any changes within the network. You can run almost any protocol above batman advanced, prominent examples are: IPv4, IPv6, DHCP, IPX.

Batman advanced was implemented as a Linux kernel driver to reduce the overhead to a minimum. It does not depend on any (other) network driver, and can be used on wifi as well as ethernet lan, vpn, etc ... (anything with ethernet-style layer 2 ). ${ }^{67}$

Instructions are given there, among others e.g. on how to load the batmanadv module into the kernel. This is done with insmod ${ }^{68}$. (A simple batman-adv won't do, nor will man batman-adv return anything.)

\section{Obstacles}

The obstacle presented by much of these technologies are habits and convenience (or lack thereof), a human factor, that is probably the biggest weakness of any system's modification. A user A may have recognized their benefits, but all of user A's network is made of people unwilling to switch to new technologies, or change their habits (in any major way).

Another obstacle, also human, is the lack of their teachings in academic computer settings : in textbooks and classes. "The systems, both human and computer".

Lastly,) the documentation for these various projects is not the best. This is an obstacle that ideally should not exist - but as most human factors, which turn out to be the most important in a lot of technological topics, contrary to intuition and common presentations, they do.

Another human factor is that such projects (often if not always?) necessitate the cooperation of many people, with all the advantages and disadvantages, and strengths and complications/weaknesses, of such systems.

\footnotetext{
${ }^{67}$ https://www.kernel.org/doc/html/v4.17/networking/batman-adv.html

68 "insmod - Simple program to insert a module into the Linux Kernel" (Man pages).
} 
The Freedom Tower by the Free Network Foundation is not the first of its kind ${ }^{69}$. But, their great strength, the way we see it, was the way they were able to package what is both an idea, and a powerful one, and a technical matter - including but not limited to building a practical, well laid-out, transportable encasing, showcasing their work to a vast public (e.g. documentary $^{70}$ ), and providing some form of somewhat accessible written documentation (report). ${ }^{71}$

\footnotetext{
${ }^{69}$ In the search for antecedents, an almost infinite activity, one could always go back to amateur radio. But, in this case, Freifunk is - if not the most - one of the most important references (expressed in their report in various ways, we believe).

${ }^{70}$ In this case, the 30-minute short documentary film was produced by Motherboard, part of Vice (Vice Media), dedicated to technology. The video, made available for free on a popular user-contributed video platform, has over half a million views currently.

${ }^{71}$ In the opinion of this author, still, and always, the Freedom Tower has a distinct urban quality to it, that, as the documentary demonstrates, shot in various parts of New York, makes them easily deployable, they can be sprung in a few minutes, and is attached a philosophicalpolitical meaning that resonates with many people (the documentary demonstrates how this has led them to receive support, financial and otherwise, or funding from a variety of sources and places). But, attaching the Freedom specifically to New York, or Zuccotti Park, or the Occupy Wall Street movement, is a mistake. It was just an experiment... "Through its offshoot KC Freedom Network, the FNF worked with area digital inclusion organization Connecting for Good to establish free networks serving more than 600 residences in the low-income housing developments of Juniper Gardens and Rosedale Ridge, both in Kansas City, Kansas." https: //www.kcdigitaldrive.org/project/free-network-foundation/. The pairings of the likes of the promoters of the Freedom Tower remind of historical characteristics of revolutionary figures, or movements (e.g. Marx and Engels' roots in the upper-middle, upper classes.) With some these social origins mean they get to go through a rebellious stage, from the place of security afforded to them by their background, on which they may then look back either fondly or not-so-fondly in middle age, with others these causes can represent far more reaching and lasting engagements.
} 


\section{Conclusion}

The Freedom Tower was presented in a series or original research notes. Shortcomings of Networks and Networking textbooks were discussed in this context.

There can be no good conclusion for such a work : the Web and Internet need "champions" who will protect them according to Berners-Lee - may they find useful information here, and elsewhere, alternatives, or their successors.

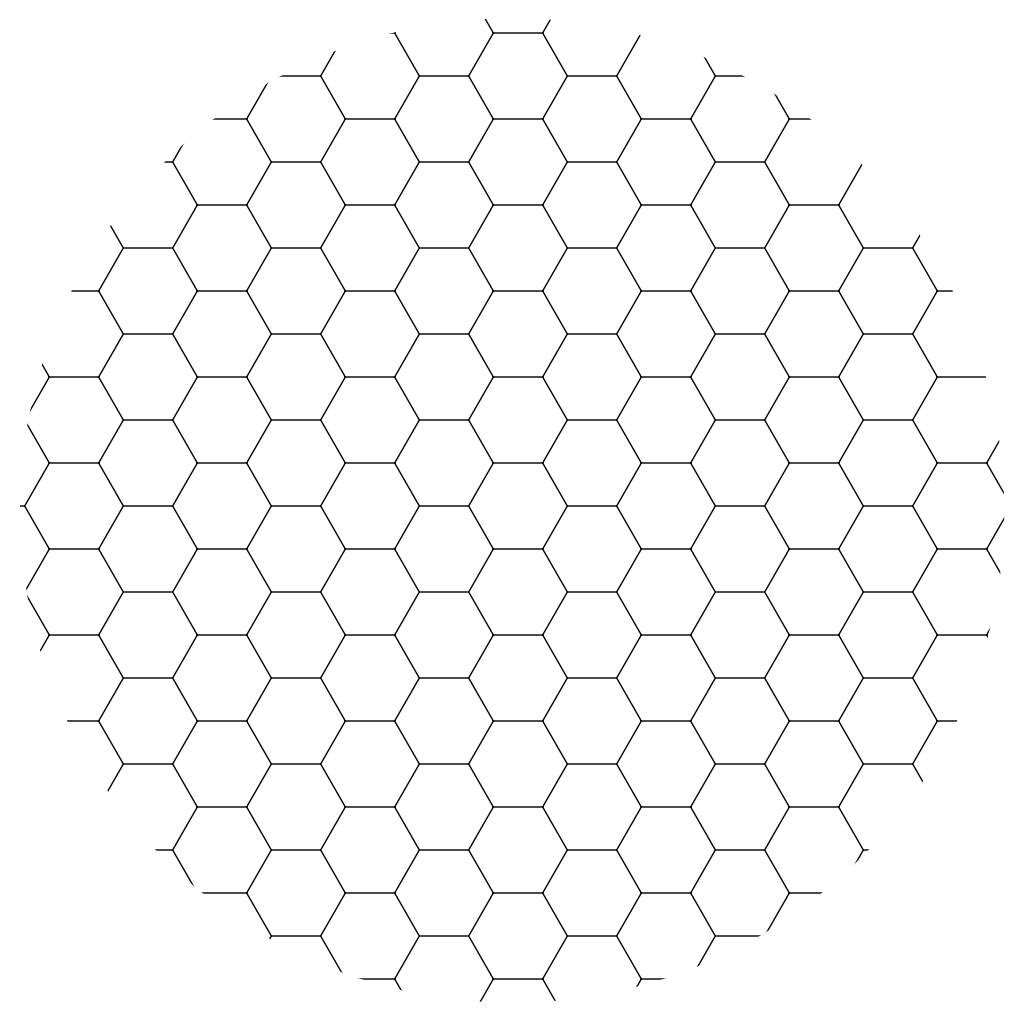




\section{Bibliography}

airberry. 2012. "Introduction to Mesh Networks". https://web.archive.org/ web/20130811032810/http://airberry.com/downloads/airberry_Whitepaper _ EN_02_Wireless_Mesh.pdf

Berkman Klein Center for Internet \& Society. 2012. "How Mesh Networks Work". https://www youtube. com/watch?v=tYLU755T6_I

Cook, Gordon. 2012. "Peer to Peer User Owned Communications Infrastructure". Cook Report on Internet Protocol. March April (Part 2). http://www . phibetaiota.net/wp-content/uploads/2012/04/2012-04-13-CookReport-Peer-to-Peer-Freedom-Tower.pdf

Comer, Douglas. 2015. Computer Networks and Internets. Upper Saddle River, NJ : Pearson Education.

Hu, Elise. 2014. "How Hong Kong Protesters Are Connecting, Without Cell Or Wi-Fi Networks." NPR, 29/09. https ://www.npr.org/sections/alltechconsidered/ 2014/09/29/352476454/how-hong-kong-protesters-are-connecting-withoutcell-or-wi-fi-networks

Huang, Andrew. 2018. "Making \& Breaking Hardware: Origins of the Hacker Ethos". https://www. youtube. com/watch?v=HwkbbVoQOxw

Kurose, James and Ross, Keith. 2017. Computer Networking: A Top-Down Approach. Harlow : Pearson Education.

Motherboard. 2012. Free the Network: Hackers Take Back the Web. https: //www . youtube. com/watch?v=Fx93WJPCCGs.

Tanenbaum, Andrew. 2003. Computer Networks. Upper Saddle River, NJ : Pearson Education.

Tanenbaum, Andrew and Wetherall, David. 2011. Computer Networks. Upper Saddle River, NJ : Pearson Education. 


\section{Appendix}

- Freedom Tower enclosure (better view).

- Freedom Tower enclosure (more primitive version).

- Freedom Tower (pole) (shown in the outside). 


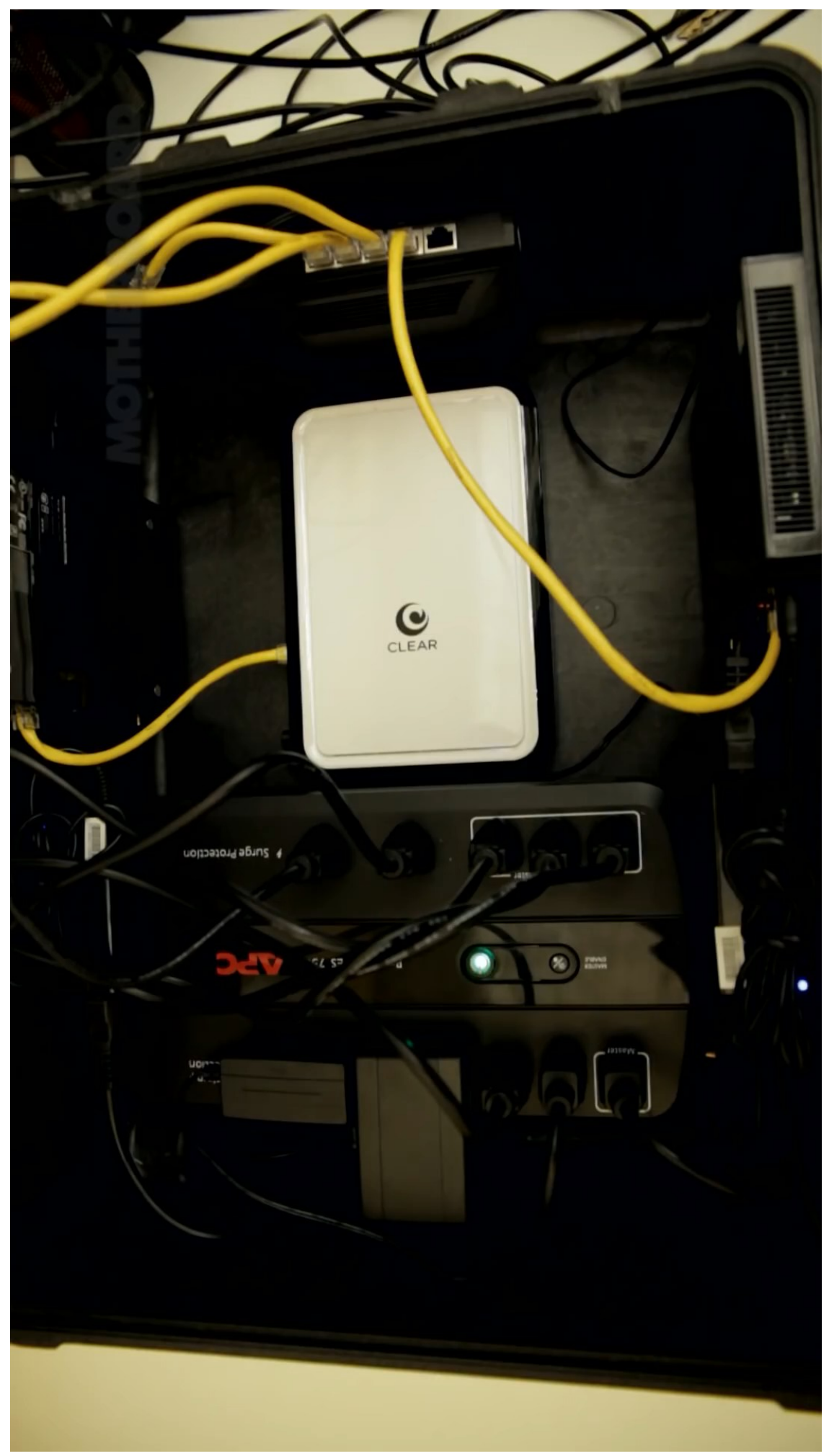

Figure 6: Freedom Tower enclosure (better view) (Motherboard 2012). 


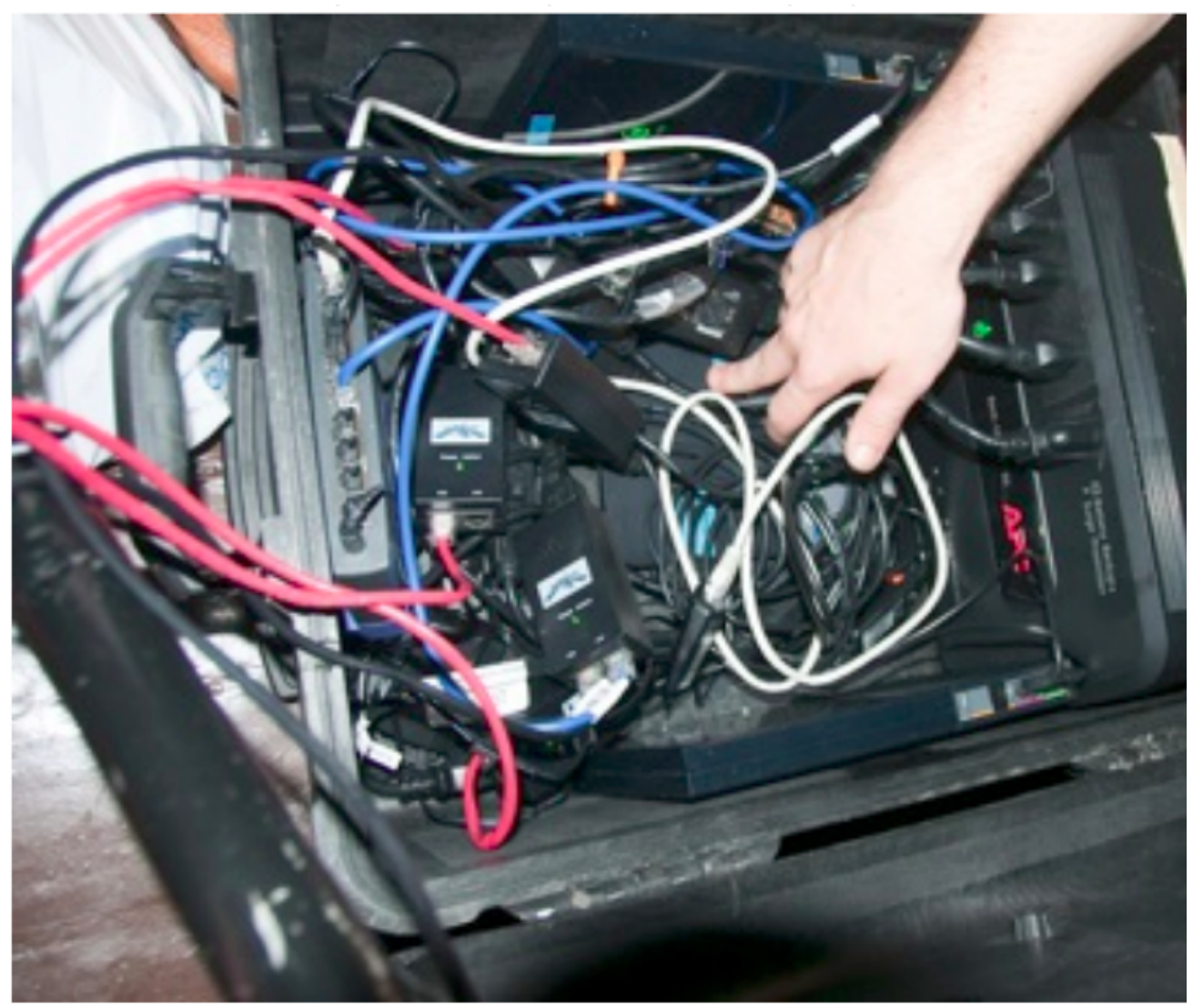

Figure 7: Freedom Tower enclosure (more primitive version) (Cook 2012). 


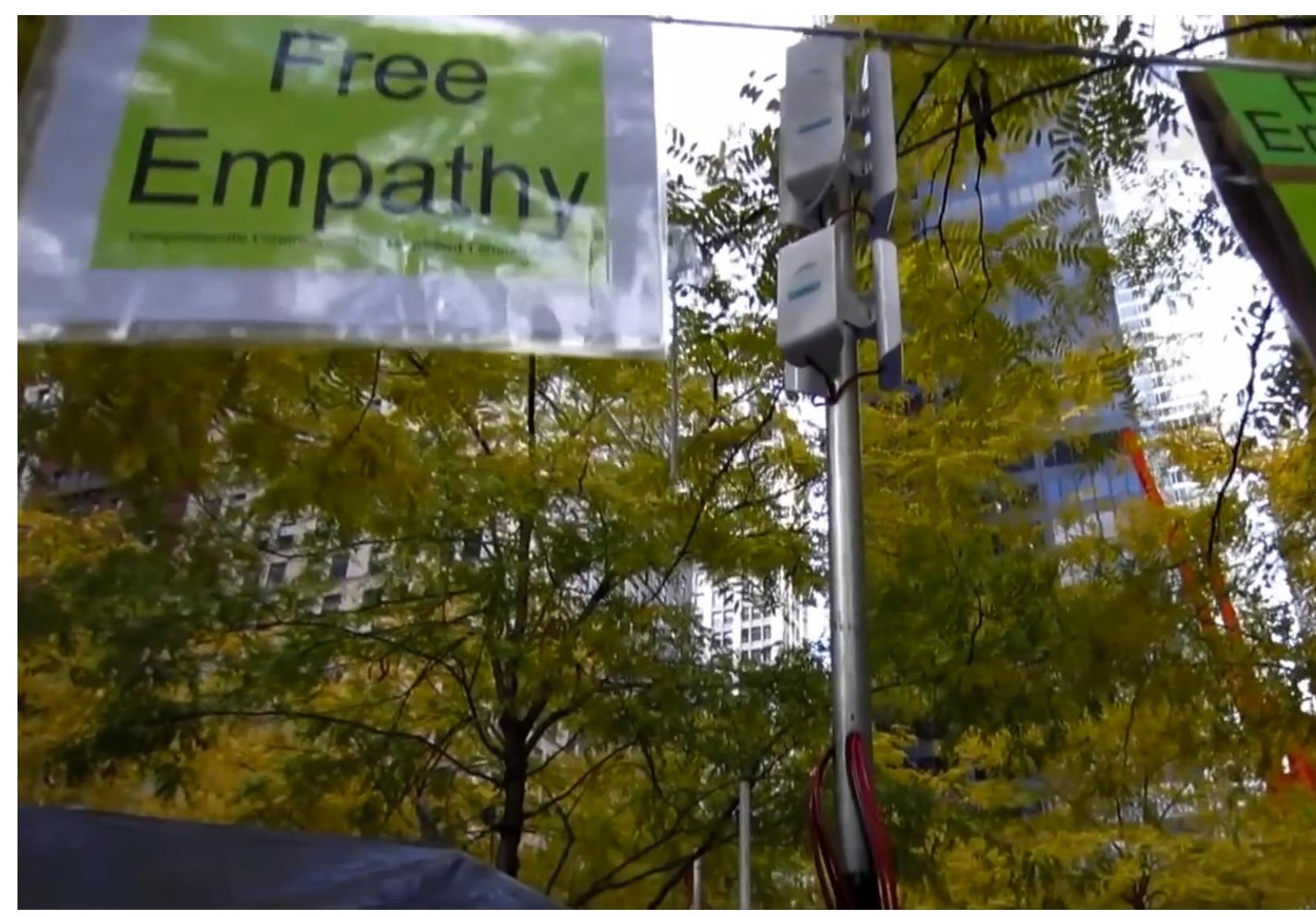

Figure 8: Freedom Tower (pole) (shown in the outside) (Motherboard 2012). 